\title{
Research on Speed Optimization Strategy of Hybrid Electric Vehicle Queue Based on Particle Swarm Optimization
}

\author{
Shaohua Wang $\mathbb{D}^{1},{ }^{1}$ Chengquan Yu, ${ }^{1}$ Dehua Shi, ${ }^{1,2}$ and Xiaoqiang Sun ${ }^{1,2}$ \\ ${ }^{1}$ School of Automobile and Traffic Engineering, Jiangsu University, Zhenjiang 212013, China \\ ${ }^{2}$ Automotive Engineering Research Institute, Jiangsu University, Zhenjiang 212013, China \\ Correspondence should be addressed to Shaohua Wang; shwang@ujs.edu.cn
}

Received 14 May 2018; Accepted 10 October 2018; Published 22 October 2018

Academic Editor: Jürgen Pannek

Copyright (C) 2018 Shaohua Wang et al. This is an open access article distributed under the Creative Commons Attribution License, which permits unrestricted use, distribution, and reproduction in any medium, provided the original work is properly cited.

\begin{abstract}
Traffic lights intersections are common in cities and have an impact on the energy consumption of vehicles, so it is significant to optimize the velocities of vehicles in urban road conditions. The novel speed optimization strategy for hybrid electric vehicle (HEV) queue that helps reduce fuel consumption and improve traffic efficiency is presented in this paper, where real-world traffic signal information is used to construct the research scenario. The initial values of the target velocities are obtained based on the signal phase and timing (SPAT). Then the particle swarm optimization (PSO) algorithm is used to solve the nonlinear constrained problem and obtain the optimal target velocities based on vehicle to vehicle communication (V2V) and vehicle to infrastructure communication (V2I). The lower controller, which applies rule based control strategy, is designed to split the power of the engine and two electric motors in a power split HEV, which is quite promising because of its advantages in fuel economy. Simulation results demonstrate the superior performance of the proposed strategy in reducing fuel consumption of the HEV queue and improving traffic smoothness.
\end{abstract}

\section{Introduction}

With rapid growth of car ownership, the problem of excessive energy consumption and environmental pollution is becoming more and more serious, and transportation accounts for a large part of energy consumption and greenhouse gas emissions in the world [1]. Energy issues and environmental issues have become the major themes of the automotive industry in the 21st century [2]. Many researchers have investigated the problems from different angles, such as human factors and improvement of vehicle or engine design [3]. As for human factors, the main consideration is the control of the driving behavior, that is, speed optimization of vehicles.

Furthermore, the intelligent transportation system (ITS) and connected vehicle (CV) technology have developed rapidly in recent years. In 2015, the first connected vehicle in Europe has hit the road. It is predicted that the popularity of the CV technology in new vehicles will reach 70\% in 2027 [4]. So measurements become available and speed optimization based on traffic information has become easier to be achieved. In the ITS, through communicating with other vehicles and traffic infrastructure, any vehicle can obtain traffic information and information about vehicles around, including traffic signal ahead, speed limits, vehicle speeds, positions, rates of acceleration, and deceleration, so as to plan a reasonable speed to avoid red light idling and collisions (i.e., vehicle speed is optimized directly by using traffic information).

Many studies have confirmed that vehicle speed has a great influence on fuel consumption and fuel economy can be improved by speed optimization [5-8]. Giovanni et al. computed optimal velocity profiles for a vehicle in an urban traffic network [5]. He et al. presented a multistage optimal control approach considering vehicle queue passing through multiple intersections [6]. In [7], the authors planned vehicle speed to reduce fuel consumption and emissions by using V2V communication and V2I communication. However, these papers are aimed at traditional fuel vehicles.

Hybrid electric vehicles (HEVs) have been under the spotlight because of their low fuel consumption and lowemission characteristics. HEVs demonstrate superior advantages under urban driving conditions, including stop-andgo patterns and frequent acceleration/deceleration. They 
have gradually entered the industrialization stage in the last decade. Therefore some researches have been done about speed optimization of HEV in the ITS in recent years. The researchers at Clemson University and University of Minnesota carried out a series of studies on HEVs with the ITS [9-12]. In [11], the authors further minimized the energy consumption of plug-in HEVs (PHEVs) through V2I communication. The optimization strategies reduced energy consumption by $60-76 \%$. A speed optimization method based on genetic algorithm for a HEV that passes through multiple intersections is proposed by Yugong Luo et al. [13]. Traffic signal information is taken into the power-split control strategy of vehicle. The average fuel consumption and the average passing time of the HEV applying the optimal speed advisory strategy are decreased. In [14], researchers present an optimal controller for a HEV to maximize fuel efficiency by using $\mathrm{CV}$ technology, unlike the general powersplit control strategy for HEVs, which only focus on the optimization of the torque distribution between the engine and the electric motor. The controller optimizes vehicle speed and power-split together. Besides, some authors have taken into account the road slope information, which optimized not only velocity, but also the battery state of charge (SOC) $[15,16]$.

However, most of the studies in fuel economic control of the HEV consider single vehicle scenario and do not consider vehicle queue. Although some consider the HEV queue, traffic light information is not taken into account [16]. There are few studies on the speed optimization of the HEV queue in the ITS, taking into account traffic light information. In this study, we propose a speed optimization strategy of $\mathrm{HEV}$ queue considering traffic light information to improve the performance of the group of connected HEVs in urban road conditions.

Aiming to minimize vehicle average energy consumption and red light idling, we use PSO algorithm to optimize the velocities of the HEVs combining the actual traffic signal lights and speed limits on a section of practical urban road. The optimal target velocity of each vehicle that we obtain is a comprehensive balance among mobility, fuel economy, safety, and comfort. The balance among them is realized via weighting factors. In addition, the lower controller is designed. It is the energy management control level, which tracks the optimal target velocity, and controls the power split between the engine and the electric motors by using rule based control strategy.

The rest of this paper is organized as follows. Section 2 describes the collection results of traffic information and the setting of operation environment of the HEV queue. Section 3 provides the description of the control structure. In Section 4, the optimization problem solution and the design of lower controller are presented. Section 5 is simulation and analysis of simulation results. Finally, Section 6 summarizes the main conclusions.

\section{Operation Environment of the HEV Queue}

In the study, the HEV queue passing through a series of intersections is studied. The scenario of the research is constructed with information of a practical urban road, as shown in Figure 1. The main features are as follows:

(1) The queue consists of six HEVs, which have the same type.

(2) There is no lane changing and overtaking.

(3) The vehicles run in a single direction in vehicle networking environment and pass through multiple intersections.

(4) The effects of nonmotorized lanes and pedestrians are not considered.

(5) Each vehicle is equipped with a communication device that receives signals from the vehicles ahead and behind, traffic lights, and other signal transmitting devices within a certain distance.

In addition, we suppose that the direction of the vehicles is the positive and the coordinate origin is in the opposite direction of the vehicles. The initial locations of the first to sixth vehicles are assumed to be $80 \mathrm{~m}, 70 \mathrm{~m}, 50 \mathrm{~m}, 42 \mathrm{~m}, 30 \mathrm{~m}$, and $16 \mathrm{~m}$, while the initial velocities of them are $10 \mathrm{~m} / \mathrm{s}$.

In this study, traffic lights and other information are collected, according to which the operation environment of the HEV queue is set up. The total distance is $5161 \mathrm{~m}$. The selected road with a speed limit of $70 \mathrm{~km} / \mathrm{h}$ and collected information are shown in Figure 2 and Table 1, respectively. There are eleven traffic lights on this section of the road. Because yellow light acts like red light, yellow light duration is counted in red light duration. According to the instant at which the first red light starts at each intersection, which is shown in Table 1, the time interval between each traffic light can be known.

\section{Control Structure}

In this section, the structure of the speed optimization controller and the lower controller is presented in Figure 3. The control structure is based on the assumption that the vehicles are equipped with $\mathrm{V} 2 \mathrm{~V}$ and V2I communication device. It is assumed that there are no communication issues such as delay and data packet loss. The speed optimization controller integrates the PSO algorithm to obtain the optimal target velocity of each vehicle based on the traffic information, including traffic signal, speed limits, and the preceding vehicle's information. The velocity of each vehicle is optimized to achieve the control objectives, including minimizing fuel consumption, ensuring safety, maximizing mobility, and comfort, under the constraints of traffic rules.

The lower controller integrates energy management algorithm. After it receives the control signal, the optimal torques of the engine and the electric motors at the moment are obtained by the control algorithm. Then the optimal control instructions are sent to the engine controller and the motor controllers. Each controller controls the corresponding component according to the received control instructions and the actual output of the components is fed back to the lower controller for closed-loop correction. 


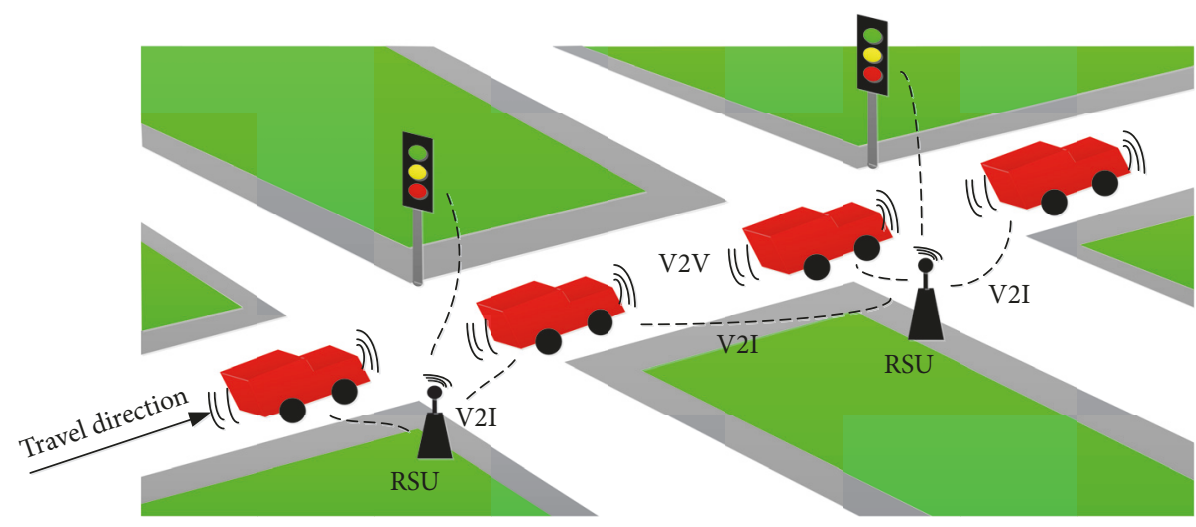

FIGURE 1: Schematic of the scenario.

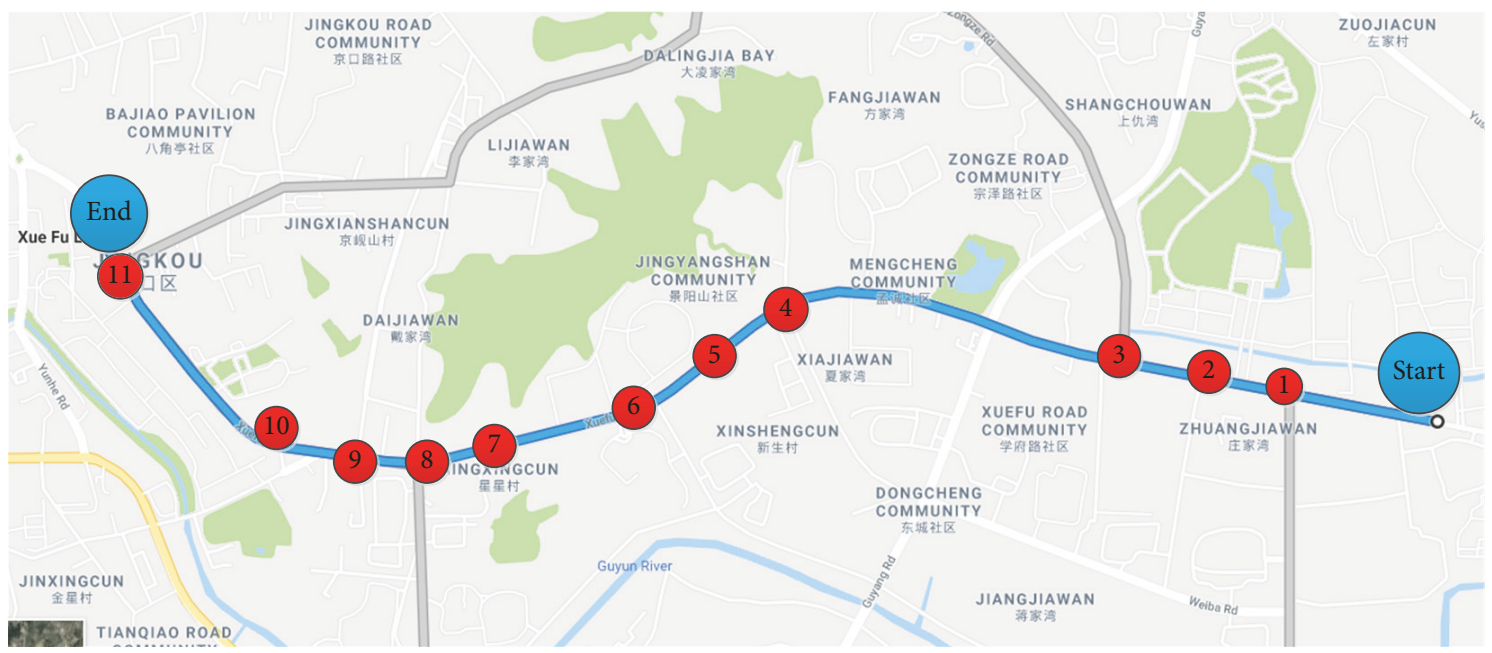

FIGURE 2: The selected road.

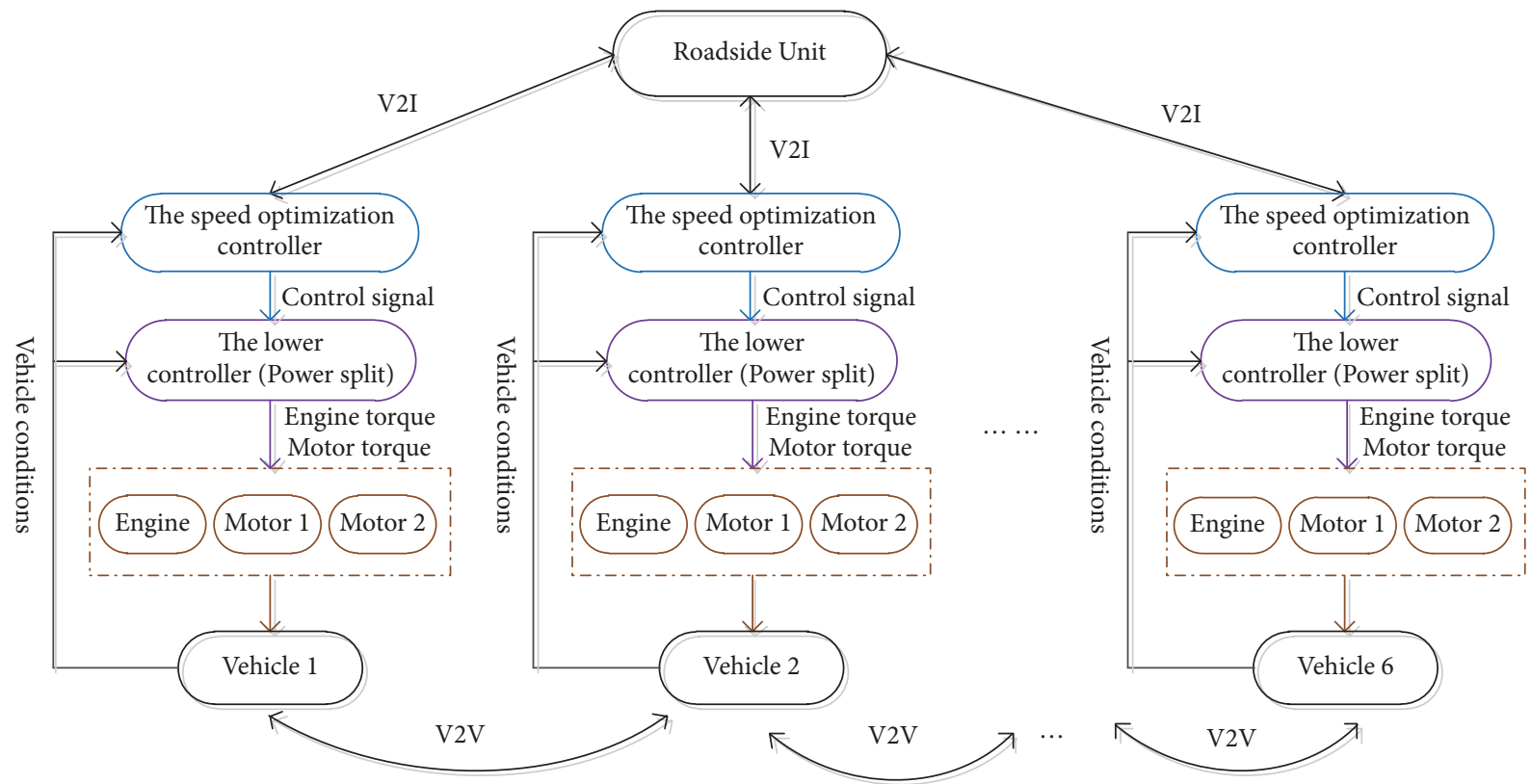

FIGURE 3: Schematic of hierarchical control. 
TABLE 1: The collected traffic information.

\begin{tabular}{|c|c|c|c|c|}
\hline $\begin{array}{l}\text { Intersection } \\
\text { no. }\end{array}$ & $\begin{array}{l}\text { Red light } \\
\text { phase(s) }\end{array}$ & $\begin{array}{c}\text { Green light } \\
\text { phase(s) }\end{array}$ & $\begin{array}{l}\text { The instant at } \\
\text { which the first } \\
\text { red light starts } \\
\text { (s) }\end{array}$ & $\begin{array}{c}\text { Distance from } \\
\text { the starting } \\
\text { point }(\mathrm{m})\end{array}$ \\
\hline 1 & 51 & 54 & 0 & 589 \\
\hline 2 & 29 & 103 & 41 & 910 \\
\hline 3 & 98 & 38 & 15 & 1669 \\
\hline 4 & 44 & 92 & 55 & 2406 \\
\hline 5 & 48 & 88 & 120 & 2739 \\
\hline 6 & 58 & 78 & 59 & 3104 \\
\hline 7 & 44 & 105 & 96 & 3628 \\
\hline 8 & 82 & 68 & 34 & 3829 \\
\hline 9 & 89 & 60 & 50 & 4046 \\
\hline 10 & 69 & 81 & 81 & 4280 \\
\hline 11 & 91 & 45 & 63 & 5161 \\
\hline
\end{tabular}

\section{Speed Optimization and Lower Level Control}

4.1. Speed Optimization. The process of solving the optimal velocity of each vehicle is divided into two parts. First of all, the initial target velocity is obtained, that is, the maximum allowable speed that vehicle can pass through the intersection in the green light window. Then the PSO algorithm is used to solve the minimum value of the objective function and obtain the optimal target velocity, where fuel economy, safety, comfort, and traffic efficiency are taken into account.

4.1.1. Vehicle Dynamics and Fuel Consumption. In vehicle queue, the longitudinal dynamics model of any vehicle $m$ is as follows [17]:

$$
\begin{aligned}
\dot{x}_{m} & =f_{m}\left(x_{m}, u_{m}\right), \\
f_{m}\left(x_{m}, u_{m}\right) & =\left[\begin{array}{c}
v_{m} \\
-\frac{1}{2 M_{m}} C_{D} \rho_{a} A_{m} v_{m}^{2}-\mu g-g \theta+u_{m}
\end{array}\right],
\end{aligned}
$$

where $x_{m}=\left[s_{m}, v_{m}\right]$ is state variable and $s_{m}$ and $v_{m}$ are location and velocity of any vehicle $m . u_{m}$ is the control variable for the vehicle $m$. In this study, $u_{m}$ refers to traction or braking force per unit mass. $M_{m}, C_{D}, \rho_{a}, A_{m}, \mu, g$, and $\theta$ are the mass of the vehicle $m$, the air resistance coefficient, the air density, the frontal area of the vehicle $m$, the rolling resistance coefficient, acceleration of gravity, and the road gradient, respectively. We assume that $\theta$ is very small so $\cos (\theta)=1$ and $\sin (\theta)=\theta$ approximately.

Because fuel economy is considered when solving the optimal target velocity, a model for evaluating fuel economy should be established. The problem of solving minimum value of the energy consumption per unit distance of $n$ vehicles over a period of time is given by the following equation:

$$
\min _{u_{m}(t)} \sum_{m=1}^{n} \frac{1}{S_{m}(T)} \sum_{t=0}^{T} m_{m f u e l}(t) \Delta t
$$

$$
\begin{array}{ll}
\text { subject to } & m_{m f u e l}(t)=\frac{1}{\eta_{m e f f} H} P_{m w}(t) \\
& P_{m w}(t) \\
= & \frac{1}{2} \rho_{a} C_{D} A_{m} v_{m}(t)^{3}+\mu M_{m} g v_{m}(t) \\
& +M_{m} g \theta v_{m}(t)+M_{m} v_{m}(t) \frac{d v_{m}(t)}{d t} \\
& v_{\min } \leq v_{m}(t) \leq v_{\max } \\
& u_{m \min } \leq u_{m}(t) \leq u_{m \max } .
\end{array}
$$

In the above equation, $T$ is vehicle travel time. $S_{m}(T)$ is the distance that the $m$ th vehicle travels within a period of time T. $m_{m f u e l}(t)$ is the rate of fuel consumption of HEVs. $\eta_{\text {meff }}$ is efficiency of the path from the fuel tank to the vehicle powertrain. $H$ is the fuel lower heating value. $P_{m w}(t)$ is the required power. $v_{\max }$ and $v_{\min }$ are the maximum and minimum allowable speed on the given road, respectively. $u_{\text {min }}$ and $u_{\text {max }}$ are the minimum and maximum allowable control variable.

4.1.2. Initial Target Velocity. Vehicles can exchange information with traffic infrastructure based on V2I. A velocity range can be obtained to avoid red light idling by using traffic light information and the distance between the vehicle and the intersection ahead.

Figure 4 shows a schematic of traffic lights distributed over space-time. Suppose gij is starting time of the $j$ th green light window of the $i$ th traffic light and rij is starting time of the $j$ th red light window of the $i$ th traffic light. $d_{m}(k)$ is the distance between $s_{m}(k)$ (location of the $m$ th vehicle at instant $k$ ) and the traffic light ahead.

For example, at the initial moment, for a vehicle to pass through the first traffic light during the first green light window, its velocity should be in the interval $\left[d_{m}(0) / r 11\right.$, $d_{m}(0) / g 11$ ] [18]. And it is only feasible if this interval has a set intersection with the allowable speed interval $\left[v_{\min }, v_{\max }\right]$. If there is no set intersection, the vehicle can not pass through 


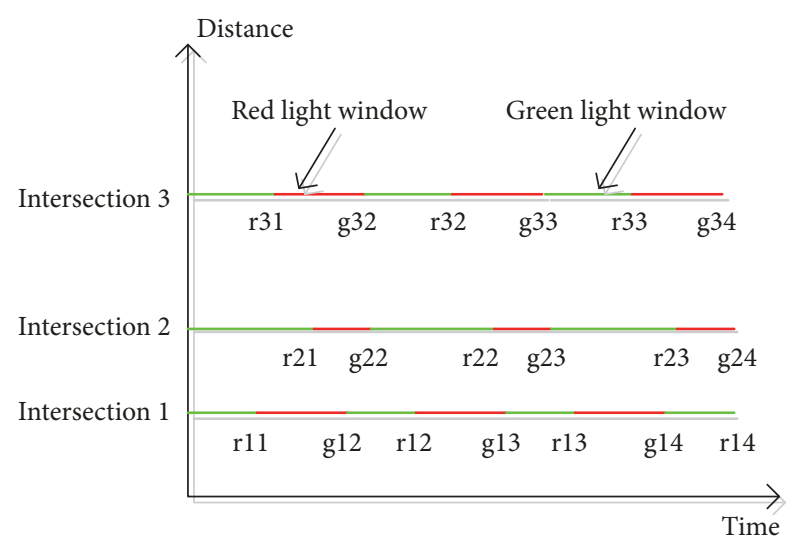

FIGURE 4: Schematic of traffic lights distributed over space-time.

the intersection during the first green light window. In that case, feasibility of passing during the next green should slow down. The process is repeated and until a green light window is available, that is, the set intersection is not empty. This set intersection is characterized as

$$
\left[\frac{d_{m}(0)}{r 1 j}, \frac{d_{m}(0)}{g 1 j}\right] \cap\left[v_{\min }, v_{\max }\right] .
$$

Of course, if the set intersection is always empty, the vehicle has to stop at the intersection.

Generally speaking, if the traffic signal ahead is red at instant $k$, the set intersection can be characterized as

$$
\left[\frac{d_{m}(k)}{r i j-k}, \frac{d_{m}(k)}{g i j-k}\right] \cap\left[v_{\min }, v_{\max }\right] .
$$

If the traffic signal ahead is green and $v_{\max } \geq d_{m}(k) / r i j$, the set intersection can be characterized as

$$
\left[\frac{d_{m}(k)}{r i j-k}, v_{\max }\right] \cap\left[v_{\min }, v_{\max }\right] .
$$

Otherwise, the set intersection can be characterized as

$$
\left[\frac{d_{m}(k)}{r i j-k}, \frac{d_{m}(k)}{g i j-k}\right] \cap\left[v_{\min }, v_{\max }\right] .
$$

The set intersection is the velocity range that ensures the vehicle would be able to avoid stopping at red light when passing through each intersection. The velocity range is characterized as $\left[v_{\text {low }}^{m}, v_{\text {high }}^{m}\right]$. So we set $v_{\text {target }}^{m}(k)$ (the initial target velocity at instant $k)=v_{\text {high }}^{m}$.

Besides, according to the velocity range, we can compute corresponding limits on control variable [19]:

$$
\begin{aligned}
& v_{m}(t) \leq v_{\text {target }}^{m}(t), \\
& v_{m}(t-1)+a \Delta t \leq v_{\text {target }}^{m}(t), \\
& \text { where } a=u_{m}(t)-\frac{1}{2 M_{m}} C_{D} \rho_{a} A_{m} v_{m}^{2}-\mu g-g \theta \\
& \quad \leq \frac{v_{\text {target }}^{m}(t)-v_{m}(t-1)}{\Delta t}, \\
& \text { similarly, } u_{m}(t)-\frac{1}{2 M_{m}} C_{D} \rho_{a} A_{m} v_{m}^{2}-\mu g-g \theta \\
& \quad \geq \frac{v_{\text {low }}^{m}(t)-v_{m}(t-1)}{\Delta t} .
\end{aligned}
$$

Thus from the above relations, we can evaluate the limits to control variable as

$$
\begin{aligned}
& \frac{v_{\text {low }}^{m}(t)-v_{m}(t-1)}{\Delta t}+\frac{1}{2 M_{m}} C_{D} \rho_{a} A_{m} v_{m}^{2}+\mu g+g \theta \\
& \leq u_{m}(t) \\
& \leq \frac{v_{\text {target }}^{m}(t)-v_{m}(t-1)}{\Delta t}+\frac{1}{2 M_{m}} C_{D} \rho_{a} A_{m} v_{m}^{2}+\mu g \\
& \quad+g \theta,
\end{aligned}
$$

such that $u_{m \min } \leq u_{m}(t) \leq u_{m \max }$.

That is to say, if the control input is in the range shown in (12), the velocity of the vehicle would be in the range $\left[v_{\text {low }}^{m}, v_{\text {high }}^{m}\right]$ and the vehicle would not meet red light when through the intersection.

4.1.3. Optimal Target Velocity. The solution of the optimal target velocity of each vehicle is a multiobjective optimization problem. In this study, mobility, fuel economy, safety, and comfort are taken into account. Correspondingly, we consider the vehicle velocity tracking, fuel consumption, the relative distance between vehicles, and the control variable. So the objective function is chosen as the sum of the weighted values of them. The objective function for solving the optimal target velocity of vehicle $m$ at instant $k$ is [20]

$$
\begin{aligned}
J_{m}(k)=\min _{u_{m}(t)} & \sum_{t=k}^{k+T-1}\left[\omega_{1}\left(v_{m}(t)-v_{\text {target }}^{m}(k)\right)^{2}+\omega_{2} \frac{m_{m f u e l}(t) \Delta t}{s_{m}(k+T-1)-s_{m}(k)}+\omega_{3}(t) R_{m n}(t)^{2}+\omega_{4} u_{m}(t)^{2}\right] \\
\text { subject to } & R_{m n}(t)=S_{0}+t_{h} v_{m}(t)-\left(s_{n}(t)-s_{m}(t)\right) \\
& \omega_{3}(t)=\alpha e^{-\beta\left(s_{n}(t)-s_{m}(t)\right)} \\
& v_{\text {low }}^{m}(t) \leq v_{m}(t) \leq v_{\text {target }}^{m}(t) .
\end{aligned}
$$




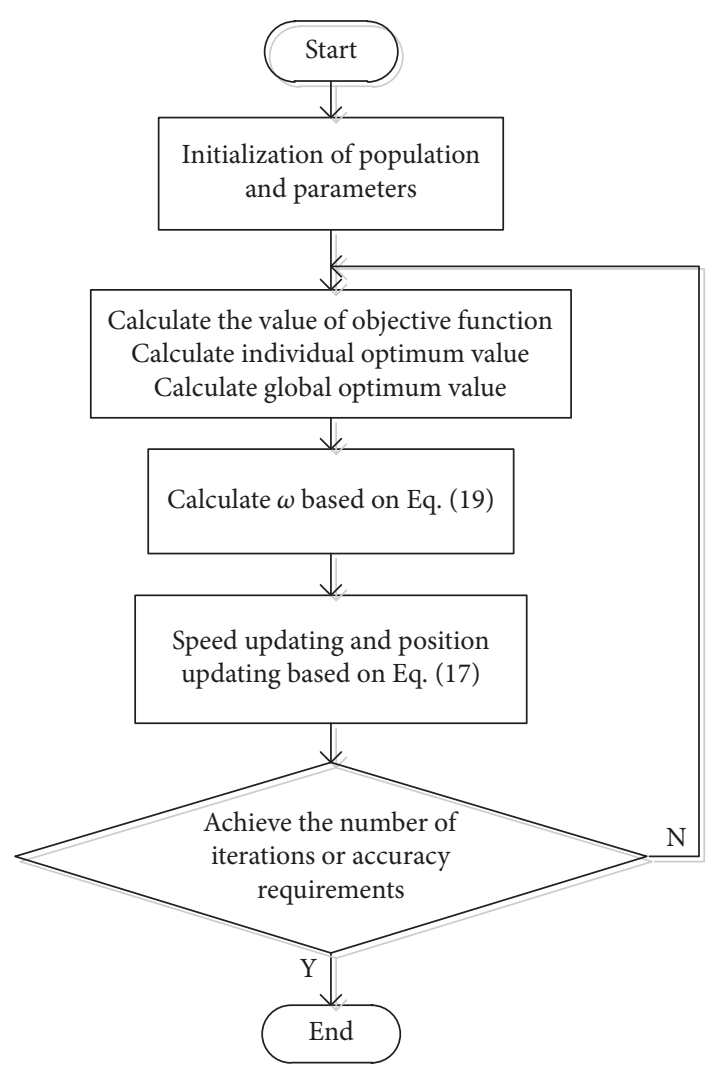

FiguRE 5: PSO process.

The range of control input $u_{m}(t)$ should be in accordance with (12). $\mathrm{S}_{0}$ and $t_{h}$ are safe distance at rest and headway time, respectively. The value of $\omega_{3}$ in (15) shows that $\omega_{3}$ increases as the relative distance between the vehicles decreases. $\omega_{1}, \omega_{2}$, and $\omega_{4}$ are constant weights.

In (14), the first term tries to make the velocity of $m$ as close as possible to the initial target velocity while the second term minimizes fuel consumption. The third term minimizes the deviation between the distance between vehicle $m$ and its preceding vehicle $\mathrm{n}$ and the safe distance. Safety and the compactness of vehicle queue are considered in this term, relying on $\mathrm{V} 2 \mathrm{~V}$. The last term minimizes the control input to avoid sudden acceleration or braking and ensure comfort.

Considering solving efficiency, the PSO algorithm is used to solve the optimal control problem as (14). PSO algorithm starts with a random solution and finds the optimal solution by iteration. Like genetic algorithm, it also uses fitness degree to evaluate the quality of solutions, but it is simpler than genetic algorithm because it has no crossover and mutation [21]. So PSO algorithm is easy to achieve with high precision and fast convergence.

The PSO algorithm design is shown in Figure 5. In an ndimensional search space, the position and speed of the $i$ th particle are

$$
\begin{aligned}
X_{i} & =\left(x_{i 1}, x_{i 2}, \ldots, x_{i n}\right), \\
V_{i} & =\left(v_{i 1}, v_{i 2}, \ldots, v_{i n}\right) .
\end{aligned}
$$

The current individual optimum value and the global optimum value are $p_{\text {best }}$ and $g_{\text {best }}$, respectively. In the each iteration, the position and speed of each particle are updated to track the two optimum values based on equation as follows:

$$
\begin{aligned}
& v_{i j}^{k+1}=\omega v_{i j}^{k}+c_{1} \cdot \operatorname{rand}_{1} \cdot\left(p_{\text {best }}-x_{i j}^{k}\right) \\
& +c_{2} \cdot \operatorname{rand}_{2} \cdot\left(g_{\text {best }}-x_{i j}^{k}\right) \\
& x_{i j}^{k+1}=x_{i j}^{k}+v_{i j}^{k+1} \\
& \text { subject to } \quad v_{i j}^{k+1}= \begin{cases}v_{p \max }, & v_{i j}^{k+1}>v_{p \max } \\
-v_{p \max }, & v_{i j}^{k+1}<-v_{p \max }\end{cases} \\
& x_{i j}^{k+1}= \begin{cases}x_{\max }, & x_{i j}^{k+1}>x_{\max } \\
x_{\min }, & x_{i j}^{k+1}<x_{\min },\end{cases}
\end{aligned}
$$

where $v_{i j}^{k+1}$ and $x_{i j}^{k+1}$ are the speed and position of the $j$ th dimensional component of the $i$ th particle at the iteration number $k+1 ; v_{p \max }$ is maximum particle velocity; $x_{\max }$ and $x_{\min }$ are the maximum position and minimum position of

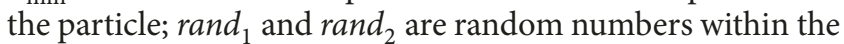
range of $[0,1] ; c_{1}$ and $c_{2}$ are the learning factors; and $\omega$ is the inertia factor.

A large inertia factor is good for jumping out of local optimum, which is convenient for global search, while a small inertia factor is beneficial to accurate search in the current search region and the convergence of the algorithm, so the weight of linear variation can be used. For the facts that the PSO algorithm has the problem of premature convergence and oscillation is easy to occur near the global optimum solution at the later stage of the PSO algorithm, the inertia weight is reduced linearly from the maximum $\omega_{\max }$ to the minimum $\omega_{\min }$. The formula that indicates $\omega$ varies with the iteration number is

$$
\omega=\omega_{\max }-\frac{k \cdot\left(\omega_{\max }-\omega_{\min }\right)}{k_{\max }},
$$

where $k$ is the current iteration number and $k_{\max }$ is the maximum number of iterations.

Considering the dynamic characteristics of the system, rolling optimization is used [22]. The first control input of the control sequence which is obtained is applied to the system. And the corresponding first velocity is the optimal target velocity at instant $k$. Then the interval is pushed forward one step and PSO algorithm is repeated. The optimal target velocity sequence is obtained by rolling optimization. The principle of rolling optimization is shown in Figure 6.

4.2. Lower Level Control. The lower controller maps the velocity profile of each vehicle obtained from the speed optimization controller to power request and calculates the torque distribution between the engine and the electric motors at each sampling time.

4.2.1. Hybrid System Configuration and Modeling. Power split HEVs have the advantages of serial and parallel HEVs. The 


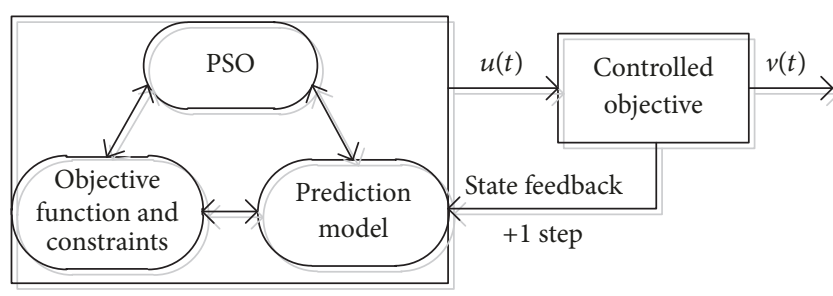

FIGURE 6: Schematic of the rolling optimization.

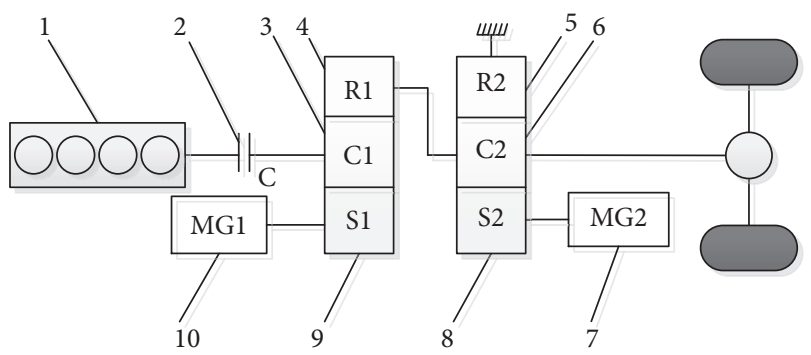

Figure 7: Structure of CHS. 1: engine, 2: locking mechanism, 3: carrier gear $\mathrm{C} 1$, 4: ring gear R1, 5: ring gear R2, 6: carrier gear C2, 7: electric motor MG2, 8: sun gear S2, 9: sun gear S1, and 10: electric motor MG1.

operation modes are more abundant and better fuel economy can be obtained with power split HEVs [23]. Now power split configuration with double planetary gear sets is also becoming more common such as THS-III. So in this study, we suppose that each vehicle is power split HEV and its hybrid system is CHS system, characterized by double planetary gear sets (PG1 and PG2) and two electric motors (MG1 and MG2); both can work as a motor or generator [24]. Structure of CHS is shown in Figure 7. Vehicle modeling is as follows:

(1) Engine model: in this study, from the view of energy distribution, the transient response of the system is neglected. As a result, the test modeling method is used to describe the behavior of the engine. The engine model can be established by a look-up table model capable of mapping engine input and output based on engine experimental data. The engine fuel rate is a function of engine torque and speed, which is described as

$$
\dot{m}_{f}=\psi_{f}\left(T_{\mathrm{E}}, \omega_{\mathrm{E}}\right)
$$

where $\dot{m}_{f}$ is the engine fuel rate, $T_{\mathrm{E}}$ is the engine torque, and $\omega_{\mathrm{E}}$ is the engine speed.

(2) Electric motor model: for energy management, the relationship between the motor demand torque and the actual torque is mainly concerned. The motor model is established by experimental data.

Then a look-up table model of motor efficiency and motor torque and speed is established. The motor efficiency and the relationship between output torque and required torque are described as

$\eta_{m}=\psi_{m}\left(T_{m}, \omega_{m}\right)$,
$T_{m}= \begin{cases}\min \left(T_{m_{\_} \text {req }}, T_{\text {max_dis }}\right), & T_{m_{\_} \text {req }}>0 \\ \max \left(T_{m_{\text {_req }}}, T_{\text {max_char }}\right), & T_{m_{\text {_req }}}<0,\end{cases}$

where $\eta_{m}$ is the motor efficiency, $T_{m}$ is the output torque of the motor, $\omega_{m}$ is the motor speed, and $T_{m_{\text {_req }}}$ is the required torque of the motor. $T_{\text {max_dis }}$ is the maximum output torque of the motor at current speed when battery is discharging, and $T_{\text {max_char }}$ is the maximum output torque of the motor at current speed when battery is charging.

(3) Battery model: the charging and discharging process of battery has complex nonlinear characteristics. The characteristic parameters involved include battery voltage, working current, internal resistance, SOC, and temperature. The modeling methods of battery mainly include RC (resistive-capacity) model, Rint (internal resistance) model, and neural network model $[25,26]$. Rint model is adopted in this paper. SOC is a main state variable when designing HEV optimal control strategy and can be calculated by

$$
\text { SOC }=-\frac{V_{\mathrm{oc}}-\sqrt{V_{\mathrm{oc}}^{2}-4 R_{\mathrm{in}} T_{m} \omega_{m} \eta_{m}^{-\operatorname{sgn}\left(T_{m}\right)}}}{2 Q_{\max } R_{\mathrm{in}}},
$$

where $V_{\text {oc }}$ is the open-circuit voltage of the battery, $Q_{\max }$ is the maximum charging capability, $R_{\text {in }}$ is the battery resistance, $T_{m}$ is the motor torque, and $\omega_{m}$ is the motor speed.

4.2.2. Energy Management Strategy. The control strategy in the lower controller is divided into two parts: mode selection and torque distribution. The mode selection module selects the proper operation modes and the torque distribution module distributes the optimal torque commands to the engine and two electric motors [27].

The lower controller chooses the appropriate operation modes based on the required torque, vehicle states, and battery SOC. In this study, a rule based control strategy is adopted. The control strategy determines the operation modes through a series of rules as follows:

(1) MG2 supplies all driving torque (mode 1), when vehicle speed is below a certain vehicle speed denoted by veh_thresh, the required torque does not exceed the maximum torque limit of MG2, and SOC is above the lower limit denoted by soc_lo.

(2) When SOC drops below soc_lo or the required torque exceeds the maximum torque limit of MG2, the engine cooperates with MG1 and MG2. Thus, the $\mathrm{HEV}$ operates in the compound driving mode (mode 2).

(3) When the required torque is negative, SOC is below the upper limit denoted by soc_hi, and vehicle speed is below $80 \mathrm{~km} / \mathrm{h}$, the compound braking mode (mode $4)$ is activated. 


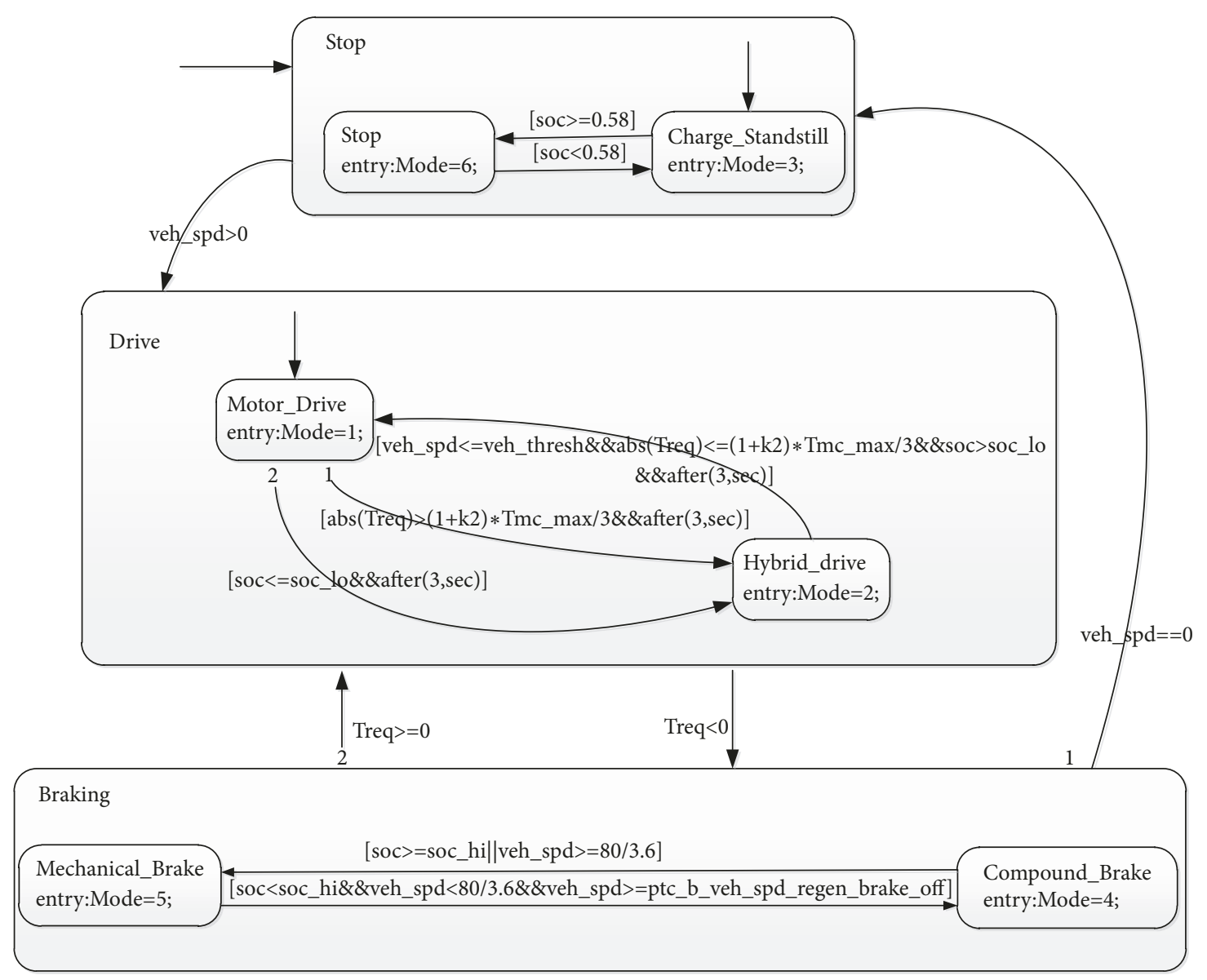

Figure 8: Stateflow chart.

(4) If the required torque is negative, when SOC reaches soc_hi or vehicle speed is above $80 \mathrm{~km} / \mathrm{h}$, the $\mathrm{HEV}$ operates in mechanical braking mode (mode 5).

(5) When the vehicle is at standstill and SOC is below 0.58 , the engine is allowed to charge the battery (mode 3 ). When SOC is above 0.58 , the engine stops charging (mode 6).

The flowchart shown in Figure 8 describes the implementation of the control strategy.

Through the analysis of the structure of power split configuration, static equation relating the torques of the engine and two electric motors can be obtained.

When the locking mechanism is locked, the engine does not provide torque, and static equation relating the torques of the two electric motors is given by

$$
T_{M}=\frac{T_{\text {out }}+K_{1} T_{\mathrm{G}}}{1+K_{2}},
$$

where $T_{\mathrm{G}}$ is torque of MG1, $T_{M}$ is torque of MG2, and $T_{\text {out }}$ is the resistance torque acting on the dynamic coupling mechanism. $K_{1}$ and $K_{2}$ are characteristic parameters of PG1 and PG2, respectively.
When the locking mechanism is disengaged, static equation relating the torques of the engine and the two electric motors is given by

$$
\begin{aligned}
& T_{\mathrm{G}}=-\frac{1}{1+K_{1}} T_{\mathrm{E}} \\
& T_{\mathrm{M}}=\frac{T_{\text {out }}}{1+K_{2}}-\frac{K_{1} T_{\mathrm{E}}}{\left(1+K_{1}\right)\left(1+K_{2}\right)},
\end{aligned}
$$

where $T_{\mathrm{E}}$ is the engine torque.

In the torque distribution module, the optimal operating line (OOL) control strategy is adopted when in the compound driving mode. The OOL control strategy can make the engine run on the optimal operating line and ensure the high efficiency of the engine. The optimal operating line of the engine is connected by the intersection points of the engine equivalent fuel consumption curve and the engine equivalent power curve.

\section{Simulation and Analysis}

Vehicle model and control strategy are built in Simulink. Some parameters of the vehicle are described in Table 2. The maximum allowable velocity on this section of the road is $70 \mathrm{~km} / \mathrm{h}$ and the minimum allowable velocity is considered 
TABLE 2: Some parameters of the vehicle.

\begin{tabular}{lc}
\hline Parameters & Value \\
\hline Vehicle mass $M_{m}$ & $1398 \mathrm{~kg}$ \\
Air resistance coefficient $C_{D}$ & 0.3 \\
Air density $\rho_{a}$ & $1.23 \mathrm{~m}^{3} / \mathrm{kg}$ \\
Frontal area $A_{m}$ & $1.746 \mathrm{~m}^{2}$ \\
Rolling resistance coefficient $(\mu)$ & 0.008 \\
Radius of wheel & $0.287 \mathrm{~m}$ \\
Ratio of the final drive & 3.93 \\
Characteristic parameter of $\mathrm{PG}_{1} K_{1}$ & 2.11 \\
Characteristic parameter of $\mathrm{PG}_{2} K_{2}$ & 2.11 \\
Engine maximum power & $54 \mathrm{~kW}$ \\
MG1 maximum power & $15 \mathrm{~kW}$ \\
MG2 maximum power & $30 \mathrm{~kW}$ \\
\hline
\end{tabular}

to be $20 \mathrm{~km} / \mathrm{h}$ in the proposed strategy. In addition, in order to verify the effect of the proposed strategy, it is compared with the strategy without speed optimization, where the target velocity is obtained only on the premise of preventing collision; that is, traffic light timing information is not used to planning speed.

Trajectories of vehicles with the two strategies are shown in Figure 9. The red solid lines represent red light windows, and the interval between the red solid lines represents green light window. As shown in Figure 9, with the two strategies, the fifth to sixth vehicles are separated from preceding vehicles at about 190 seconds, but they join in again before passing through the last intersection. With the strategy without speed optimization, the vehicles have to stop more frequently when passing through intersections, while with the proposed strategy, only at one intersection, the vehicles can not avoid stopping. The vehicles that are not separated always keep each other at an appropriate safety distance without any collision and separation, which verifies the validity of the proposed strategy.

The velocity profiles of the vehicles with the two strategies are shown in Figure 10. Under the control of the proposed strategy, vehicles only stop at one intersection during simulation time, which suggests that the vehicles have good mobility and the road has high traffic efficiency with the proposed strategy. In addition, with the proposed strategy, the change trends of velocities of 1-4 vehicles are different from those of 5-6 vehicles, which indicates the separation of vehicles. It is in agreement with the representation of Figure 9. The change time of velocities of the vehicles that are not separated is basically consistent, which shows that good communications among vehicles are given and the proposed strategy can avoid collision effectively. With the strategy without speed optimization, vehicles have to stop more frequently and the implication of changes of velocities is also in agreement with the representation of Figure 9.

The SOC curves of vehicles and responses of the engine and electric motors for vehicle 2 with the two strategies are shown in Figures 11 and 12, respectively. The SOC curves with the proposed strategy show a smoother tendency than those of the strategy without speed optimization, which indicates that the proposed strategy is more advantageous to battery than the strategy without speed optimization.

Since the ring gear of PG2 is fixed, the direction of MG2 speed is the same as the speed direction at the input side of the final drive [28]. The speed of MG2 is also proportional to the wheel speed. Consequently, when the torque of MG2 is negative, MG2 regenerates the braking energy. From the engine torque responses in Figure 12(a), it can be observed that the working time of engine with strategy without speed optimization is longer than that with the proposed strategy.

The torque and speed of MG1 are given in Figures 12(c) and 12(d), while Figures 12(e) and 12(g) describe the power of MG1 and MG2. It is obvious that the rated power of MG1 and MG2 meets the driving requirements. With the strategy without speed optimization, MG2 generates electricity in most time regions, and the time is longer than that with the proposed strategy which is in line with the phenomenon that SOC curve rises more with strategy without speed optimization.

In Table 3, comparisons between the fuel consumption and battery SOC of the vehicles for the two strategies are made. As can be seen from Table 3, fuel economy of each vehicle is improved when the proposed strategy is applied. The comparison between the strategy without speed optimization and the proposed strategy is shown in Table 4. It is observed that when the proposed strategy is adopted, the average fuel consumption is decreased by about $31.05 \%$, which means realizing economic assistant driving. It can be seen that the proposed strategy results in reduction of number of stopping instances, which shows the improvement in mobility and traffic smoothness. Thus the rationality and superiority of the proposed strategy are verified.

\section{Conclusions}

The paper proposes a novel speed optimization strategy based on the PSO algorithm for the HEV queue in the ITS, where V2V and V2I information exchange can be achieved. In this study, the basic theory and technical support are provided for the further improvement of fuel economy of HEVs. The speed optimization controller is designed to improve fuel economy, safety, comfort, and mobility of the vehicle queue. It can avoid red light idling as far as possible and collision by using the preceding vehicle's information and traffic information, including traffic signal timing phases and speed limits. Vehicle model and energy management strategy are also established to further optimize fuel economy. The benefit of the proposed strategy is significant when compared to the strategy without speed optimization. The following conclusions can be drawn based on the simulations: (i) HEVs can avoid stopping at red light as far as possible under the control of the proposed strategy. (ii) The proposed strategy is more advantageous to battery. (iii) Compared to the strategy without speed optimization, the proposed strategy can improve average fuel efficiency of HEVs by $31.05 \%$ and improve the traffic smoothness.

From the simulation process, the proposed algorithm is fast for computation and has the potential for realtime vehicle control in real-world urban set-ups. Of course, 


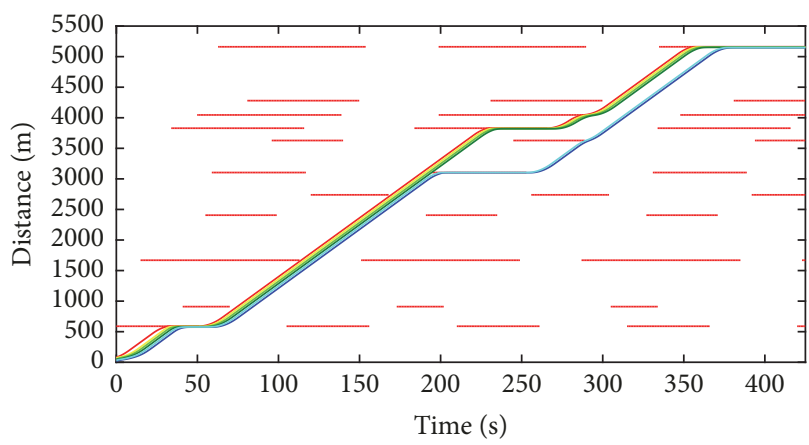

(a) Strategy without speed optimization

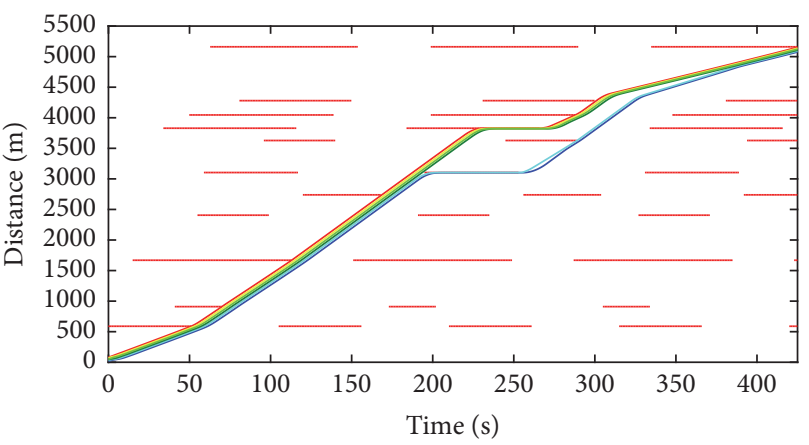

(b) Proposed strategy

Figure 9: Trajectories of vehicles.

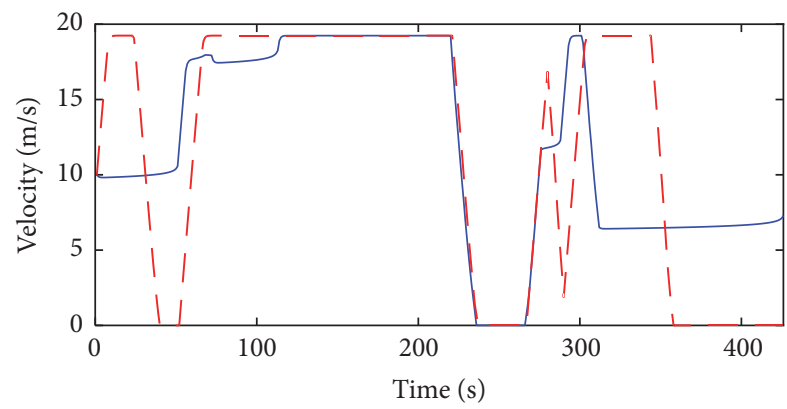

_ Proposed strategy

- - Strategy without speed optimization

(a) Vehicle 1

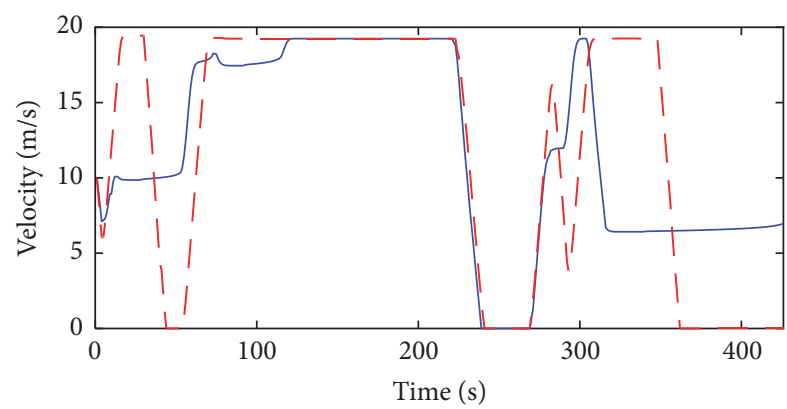

_ Proposed strategy

- - Strategy without speed optimization

(c) Vehicle 3

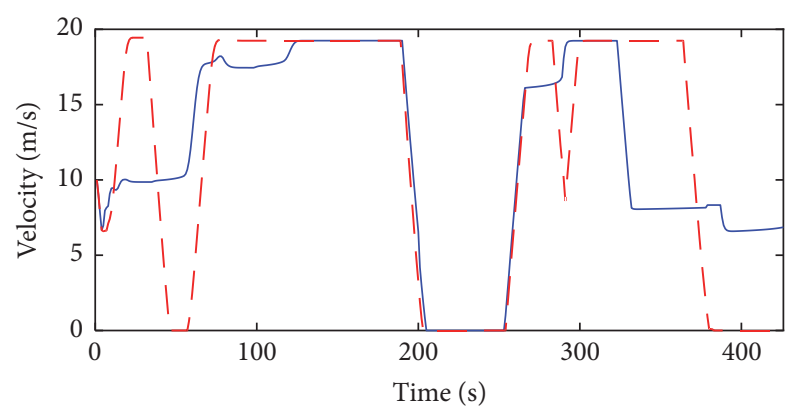

— Proposed strategy

- - Strategy without speed optimization

(e) Vehicle 5

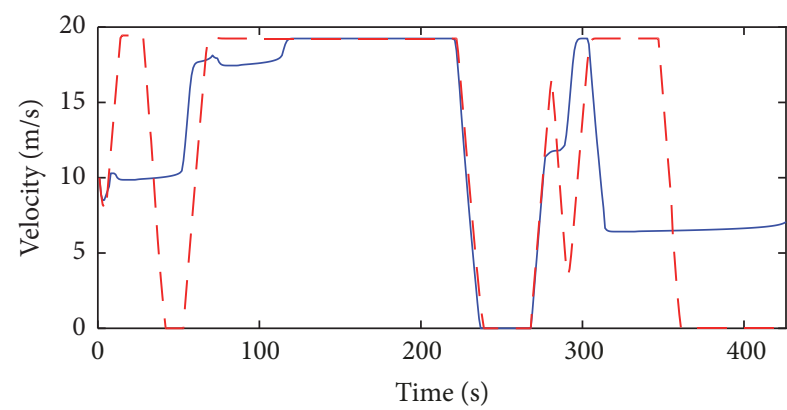

_ Proposed strategy

- - Strategy without speed optimization

(b) Vehicle 2

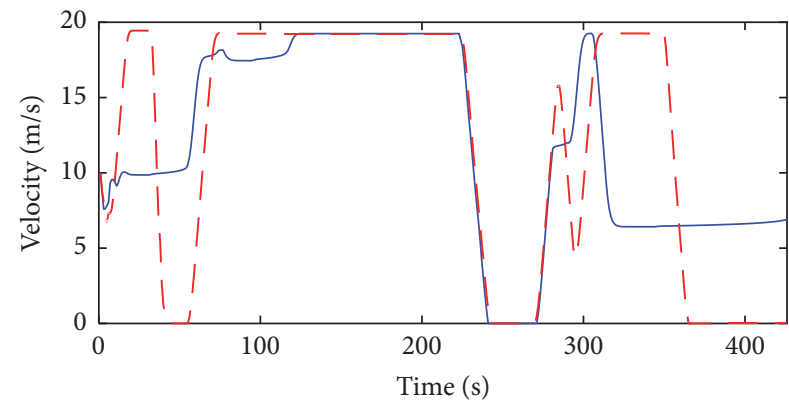

_ Proposed strategy

- - Strategy without speed optimization

(d) Vehicle 4

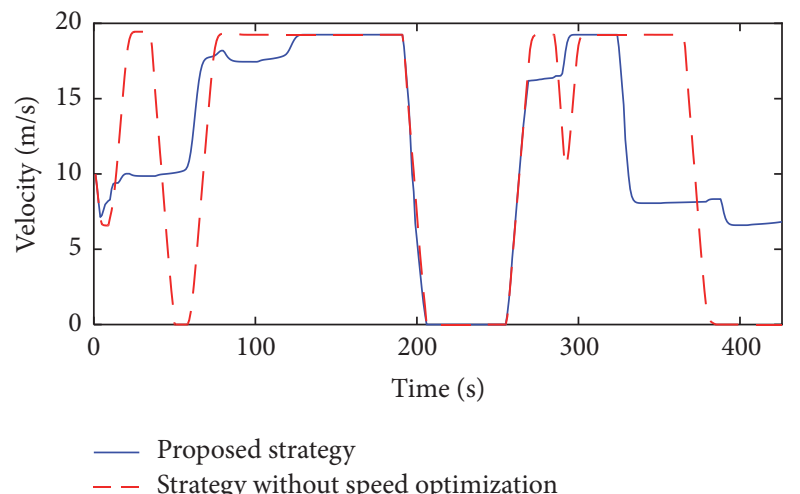

(f) Vehicle 6

FIGURE 10: Vehicle velocity profiles with the proposed strategy and the strategy without speed optimization. 


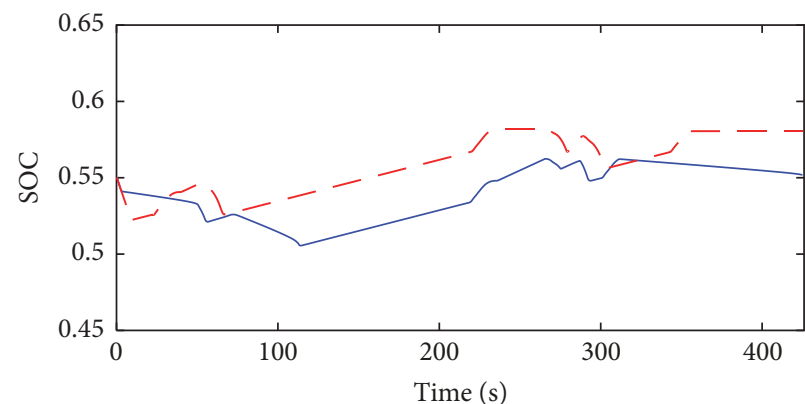

— Proposed strategy

- - Strategy without speed optimization

(a) Vehicle 1

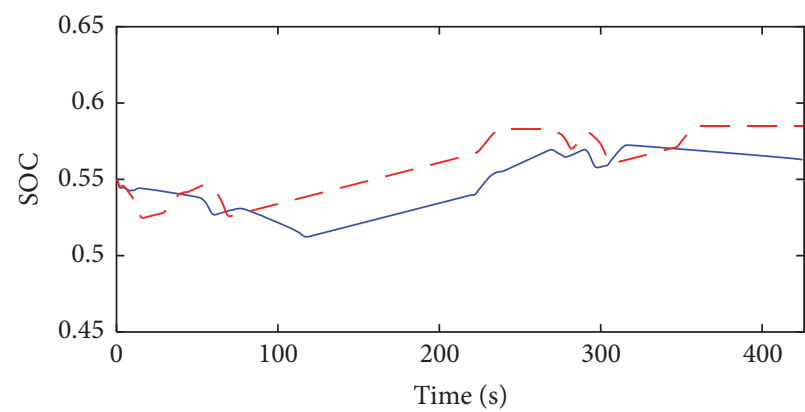

_ Proposed strategy

- - Strategy without speed optimization

(c) Vehicle 3

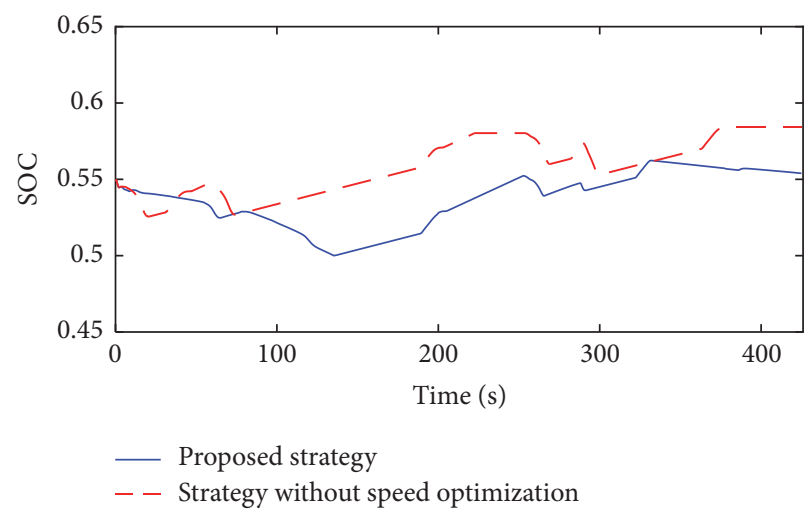

(e) Vehicle 5

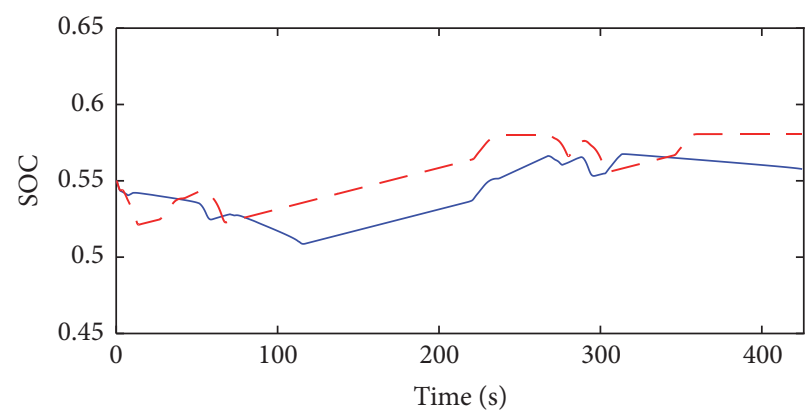

_ Proposed strategy

- - Strategy without speed optimization

(b) Vehicle 2

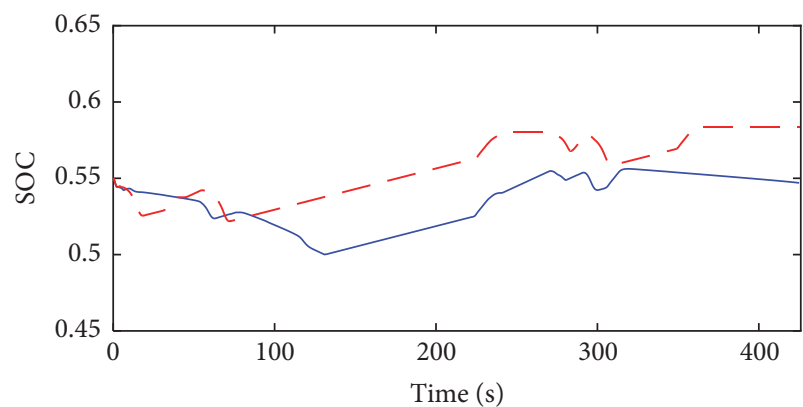

— Proposed strategy

- - Strategy without speed optimization

(d) Vehicle 4

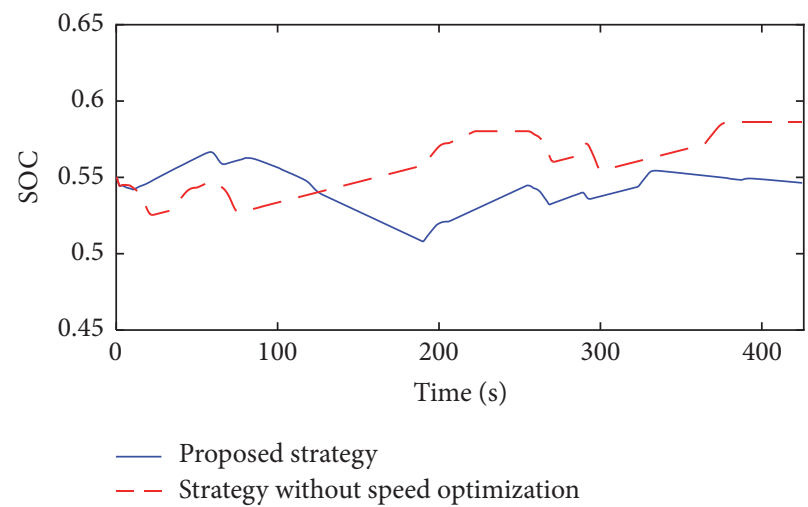

(f) Vehicle 6

FIGURE 11: Change of SOC.

we know predictive control algorithm needs to solve the optimization problem repeatedly and has a large amount of computation online, so its application is limited. Therefore, we will continue to optimize the algorithm in future research to reduce the online computation of controller and accelerate the control process without reducing the quality of control evidently. For example, the control variable is expressed as a combination of a set of primary functions, so that online optimization variables can be transformed into coefficients of a small number of primary functions. We will also study the effect of the different prediction time horizon and variable parameters will also be used to deal with the prediction time horizon and control time horizon. Furthermore, with large servers, powerful processors, advanced ITS, and other highperformance hardware devices, real-time implementation ability will be no problem.

In addition, the future research could investigate more complicated situation, such as lane changing and overtaking because vehicles usually change lanes or overtake inevitably in real-world urban set-ups. In this case, it is necessary to study vehicle transverse control. After lane changing or overtaking, the vehicle queues will regroup and the proposed strategy is still suitable for regrouped vehicle queues. Road slope can also be taken into account in subsequent studies. 


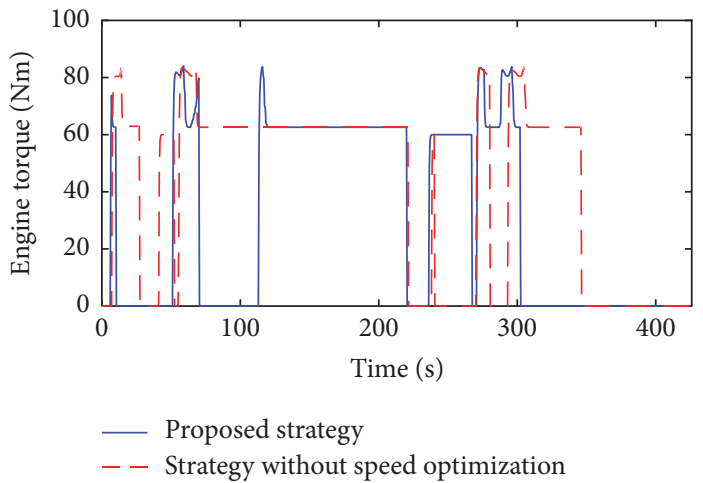

(a)

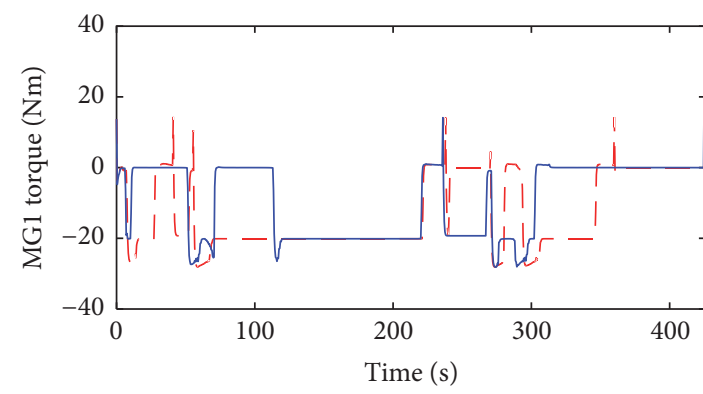

_ Proposed strategy

- - Strategy without speed optimization

(c)

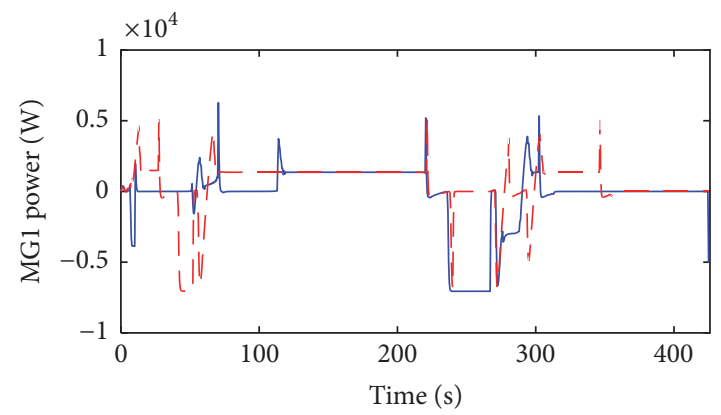

_ Proposed strategy

- - Strategy without speed optimization

(e)

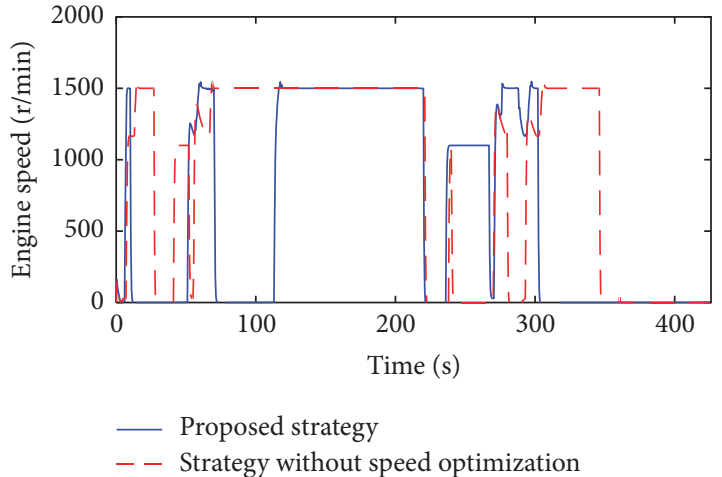

(b)

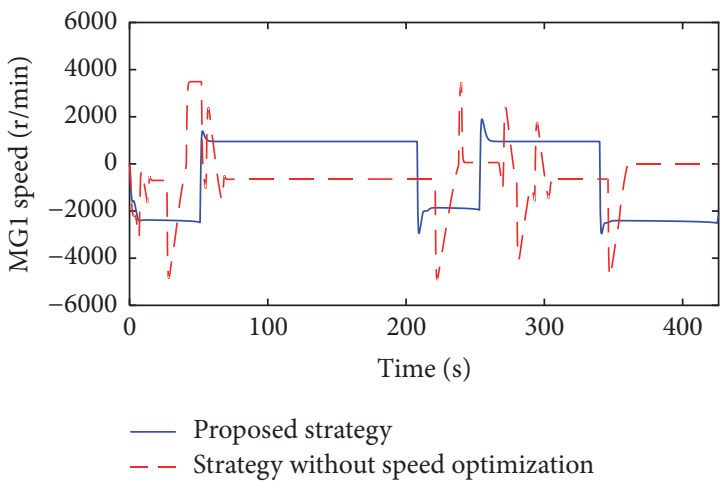

(d)

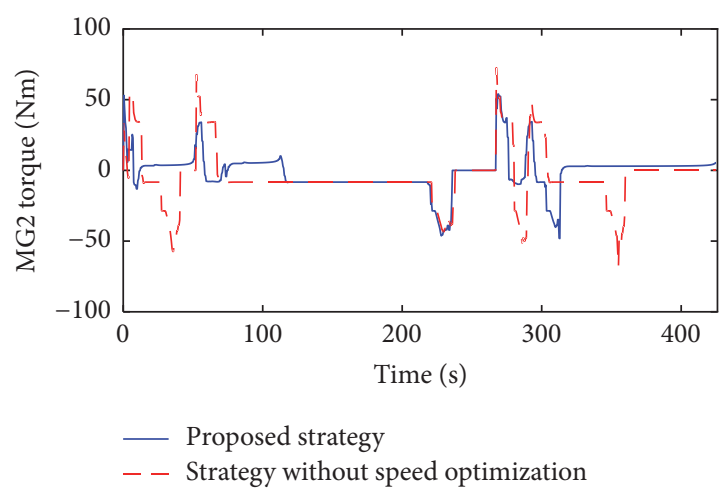

(f)

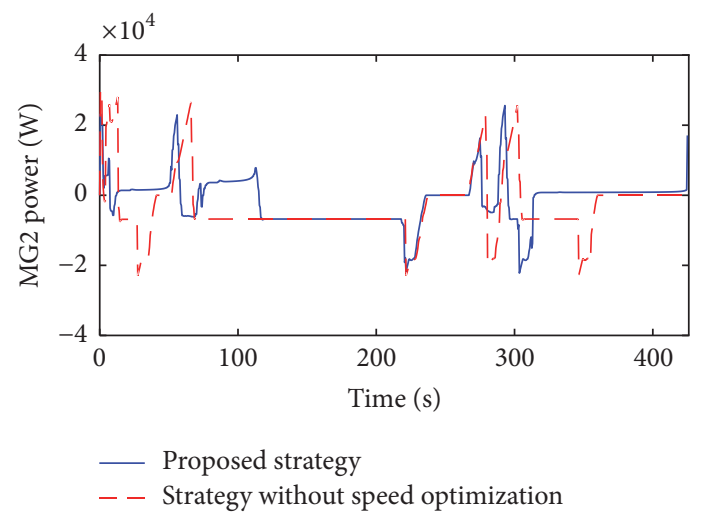

(g)

FIGURE 12: Responses of the engine and electric motors for vehicle 2. 
TABLE 3: Fuel consumption of vehicles for different strategies.

\begin{tabular}{|c|c|c|c|c|c|c|}
\hline \multirow{2}{*}{$\begin{array}{l}\text { Vehicle } \\
\text { number }\end{array}$} & \multicolumn{3}{|c|}{$\begin{array}{c}\text { Strategy without } \\
\text { speed } \\
\text { optimization }\end{array}$} & \multicolumn{3}{|c|}{$\begin{array}{l}\text { Proposed } \\
\text { strategy }\end{array}$} \\
\hline & $\begin{array}{c}\text { Engine fuel } \\
\text { consumption(g) }\end{array}$ & Final SOC & $\begin{array}{l}\text { Equivalent } \\
\text { fuel } \\
\text { consumption }(\mathrm{g})\end{array}$ & $\begin{array}{c}\text { Engine fuel } \\
\text { consumption }(\mathrm{g})\end{array}$ & Final SOC & $\begin{array}{l}\text { Equivalent } \\
\text { fuel } \\
\text { consumption }(\mathrm{g})\end{array}$ \\
\hline 1 & 168.4 & 0.580 & 168.38 & 119.3 & 0.551 & 119.29 \\
\hline 2 & 170.4 & 0.580 & 170.38 & 122.3 & 0.557 & 122.29 \\
\hline 3 & 170.2 & 0.585 & 170.18 & 123.6 & 0.562 & 123.59 \\
\hline 4 & 172.6 & 0.583 & 172.58 & 111.5 & 0.546 & 111.50 \\
\hline 5 & 176.4 & 0.584 & 176.38 & 120.0 & 0.553 & 119.99 \\
\hline 6 & 175.0 & 0.586 & 174.98 & 115.5 & 0.546 & 115.50 \\
\hline
\end{tabular}

TABLE 4: Comparison between the strategy without speed optimization and the proposed strategy.

\begin{tabular}{lcc}
\hline & $\begin{array}{c}\text { Average } \\
\text { equivalent } \\
\text { fuel }\end{array}$ & Total number of stopping instances of vehicles \\
consumption $(\mathrm{g})$ & 172.15 & 18 \\
\hline Strategy without speed optimization & 118.69 & 6 \\
Proposed strategy & & 6 \\
\hline
\end{tabular}

Through further improvement, the proposed strategy will be more adaptive and feasible for the various complexities of the real-world urban environment and can be used in automatic driving vehicles or Advanced Driver Assistance Systems (ADAS).

\section{Data Availability}

The data used to support the findings of this study are included within the article.

\section{Conflicts of Interest}

The authors declare that they have no conflicts of interest.

\section{Acknowledgments}

This work was supported by National Natural Science Foundation of China (Grant No. 51475213) and National Key R\&D Plan (Grant No. 2017YFB 0103200).

\section{References}

[1] Wei Li, Hao Li, Huixia Zhang, and Shuang Sun, "The Analysis of $\mathrm{CO}_{2}$ Emissions and Reduction Potential in China's Transport Sector," Mathematical Problems in Engineering, vol. 2016, Article ID 1043717, 12 pages, 2016.

[2] W. F. Powers and P. R. Nicastri, "Automotive vehicle control challenges in the 21st century," Control Engineering Practice, vol. 8, no. 6, pp. 605-618, 2000.

[3] S. Mendez and B. Thirouard, "Using multiple injection strategies in diesel combustion: Potential to improve emissions, noise and fuel economy trade-off in low CR engines," SAE International Journal of Fuels and Lubricants, vol. 1, no. 1, pp. 662674, 2009.
[4] G. Nelson, "U.S. regulators pave way for vehicle-to-vehicle communications, safety technology," Automotive News, 2015.

[5] G. De Nunzio, C. Canudas de Wit, P. Moulin, and D. Di Domenico, "Eco-driving in urban traffic networks using traffic signals information," International Journal of Robust and Nonlinear Control, vol. 26, no. 6, pp. 1307-1324, 2016.

[6] X. He, H. X. Liu, and X. Liu, "Optimal vehicle speed trajectory on a signalized arterial with consideration of queue," Transportation Research Part C: Emerging Technologies, vol. 61, pp. 106-120, 2015.

[7] M. Alsabaan, K. Naik, and T. Khalifa, "Optimization of fuel cost and emissions using V2V communications," IEEE Transactions on Intelligent Transportation Systems, vol. 14, no. 3, pp. 14491461, 2013.

[8] S. Mandava, K. Boriboonsomsin, and M. Barth, "Arterial velocity planning based on traffic signal information under light traffic conditions. intelligent transportation systems," in Proceedings of the 12th International IEEE Conference on Intelligent Transportation Systems, pp. 160-165, 2009.

[9] Y. He, M. Chowdhury, Y. Ma, and P. Pisu, "Merging mobility and energy vision with hybrid electric vehicles and vehicle infrastructure integration," Energy Policy, vol. 41, pp. 599-609, 2012.

[10] P. Bhavsar, Y. He, M. Chowdhury, R. Fries, and A. Shealy, "Energy Consumption Reduction Strategies for Plug-In Hybrid Electric Vehicles with Connected Vehicle Techno- logy in Urban Areas," Journal of the Transportation Research Board, vol. 2424, no. 1, pp. 29-38, 2014.

[11] Y. He, J. Rios, M. Chowdhury, P. Pisu, and P. Bhavsar, "Forward power-train energy management modeling for assessing benefits of integrating predictive traffic data into plug-in-hybrid electric vehicles," Transportation Research Part D: Transport and Environment, vol. 17, no. 3, pp. 201-207, 2012.

[12] M. A. Mohd Zulkefli, J. Zheng, Z. Sun, and H. X. Liu, "Hybrid powertrain optimization with trajectory prediction based on 
inter-vehicle-communication and vehicle-infrastructure-integration," Transportation Research Part C: Emerging Technologies, vol. 45, pp. 41-63, 2014.

[13] Y. Luo, S. Li, S. Zhang, Z. Qin, and K. Li, "Green light optimal speed advisory for hybrid electric vehicles," Mechanical Systems \& Signal Processing, vol. 87, no. 2017, pp. 30-44, 2016.

[14] J. Hu, Y. Shao, Z. Sun, M. Wang, J. Bared, and P. Huang, "Integrated optimal eco-driving on rolling terrain for hybrid electric vehicle with vehicle-infrastructure communication," Transportation Research Part C: Emerging Technologies, vol. 68, pp. 228-244, 2016.

[15] K. Yu, J. Yang, and D. Yamaguchi, "Model predictive control for hybrid vehicle ecological driving using traffic signal and road slope information," Control Theory and Technology, vol. 13, no. 1, pp. 17-28, 2015.

[16] Kaijiang Yu, Xiaozhuo Xu, Qing Liang et al., "Model Predictive Control for Connected Hybrid Electric Vehicles," Mathematical Problems in Engineering, vol. 2015, Article ID 318025, 15 pages, 2015.

[17] M. A. S. Kamal, M. Mukai, J. Murata, and T. Kawabe, "Model predictive control of vehicles on urban roads for improved fuel economy," IEEE Transactions on Control Systems Technology, vol. 21, no. 3, pp. 831-841, 2013.

[18] B. Asadi and A. Vahidi, "Predictive cruise control: utilizing upcoming traffic signal information for improving fuel economy and reducing trip time," IEEE Transactions on Control Systems Technology, vol. 19, no. 3, pp. 707-714, 2011.

[19] B. HomChaudhuri, A. Vahidi, and P. Pisu, "A fuel economic model predictive control strategy for a group of connected vehicles in urban roads," in Proceedings of the 2015 American Control Conference, ACC 2015, pp. 2741-2746, USA, July 2015.

[20] B. HomChaudhuri, R. Lin, and P. Pisu, "Hierarchical control strategies for energy management of connected hybrid electric vehicles in urban roads," Transportation Research Part C: Emerging Technologies, vol. 62, pp. 70-86, 2016.

[21] Yudong Zhang, Shuihua Wang, and Genlin Ji, "A Comprehensive Survey on Particle Swarm Optimization Algorithm and Its Applications," Mathematical Problems in Engineering, vol. 2015, Article ID 931256, 38 pages, 2015.

[22] Bin Huang, Sen Wu, Song Huang, and Xiang Fu, "Lateral Stability Control of Four-Wheel Independent Drive Electric Vehicles Based on Model Predictive Control," Mathematical Problems in Engineering, vol. 2018, Article ID 6080763, 15 pages, 2018.

[23] K. Ç. Bayindir, M. A. Gözüküçük, and A. Teke, "A comprehensive overview of hybrid electric vehicle: powertrain configurations, powertrain control techniques and electronic control units," Energy Conversion and Management, vol. 52, no. 2, pp. 1305-1313, 2011.

[24] D. Shi, P. Pisu, L. Chen, S. Wang, and R. Wang, "Control design and fuel economy investigation of power split HEV with energy regeneration of suspension," Applied Energy, vol. 182, pp. 576589, 2016.

[25] L. Xu, J. Wang, and Q. Chen, "Kalman filtering state of charge estimation for battery management system based on a stochastic fuzzy neural network battery model," Energy Conversion and Management, vol. 53, no. 1, pp. 33-39, 2012.

[26] V. H. Johnson, "Battery performance models in ADVISOR," Journal of Power Sources, vol. 110, no. 2, pp. 321-329, 2002.

[27] P. Ahmadizadeh, B. Mashadi, and D. Lodaya, "Energy management of a dual-mode powersplit powertrain based on the
Pontryagin's minimum principle," IET Intelligent Transport Systems, vol. 11, no. 9, pp. 561-571, 2017.

[28] D. Shi, S. Wang, P. Pisu, L. Chen, R. Wang, and R. Wang, "Modeling and optimal energy management of a power split hybrid electric vehicle," Science China Technological Sciences, vol. 60 , no. 5, pp. 713-725, 2017. 


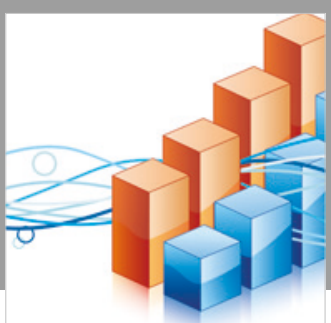

Advances in

Operations Research

\section{-n-m}
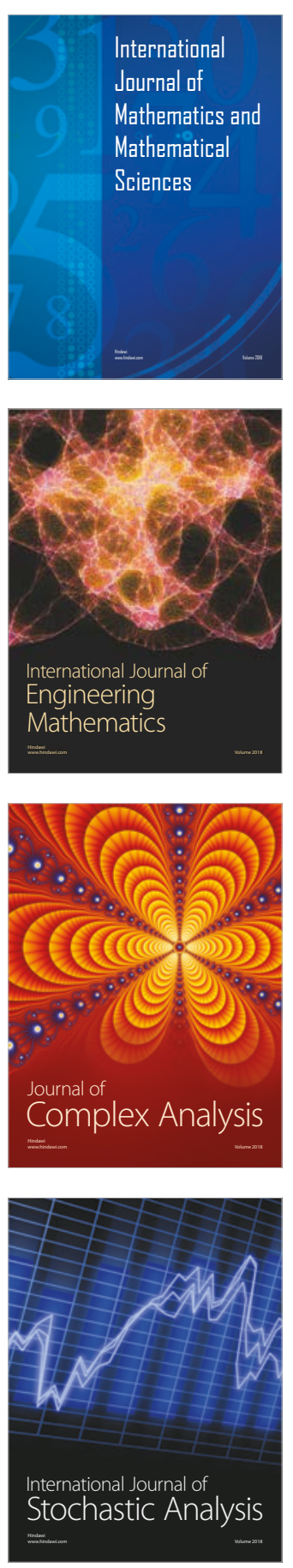
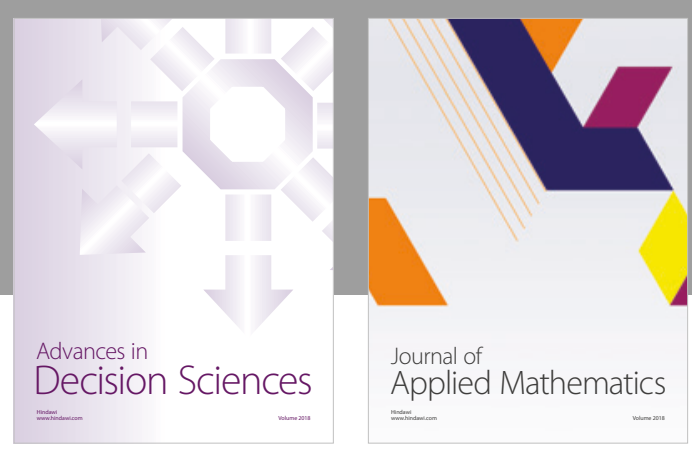

Journal of

Applied Mathematics
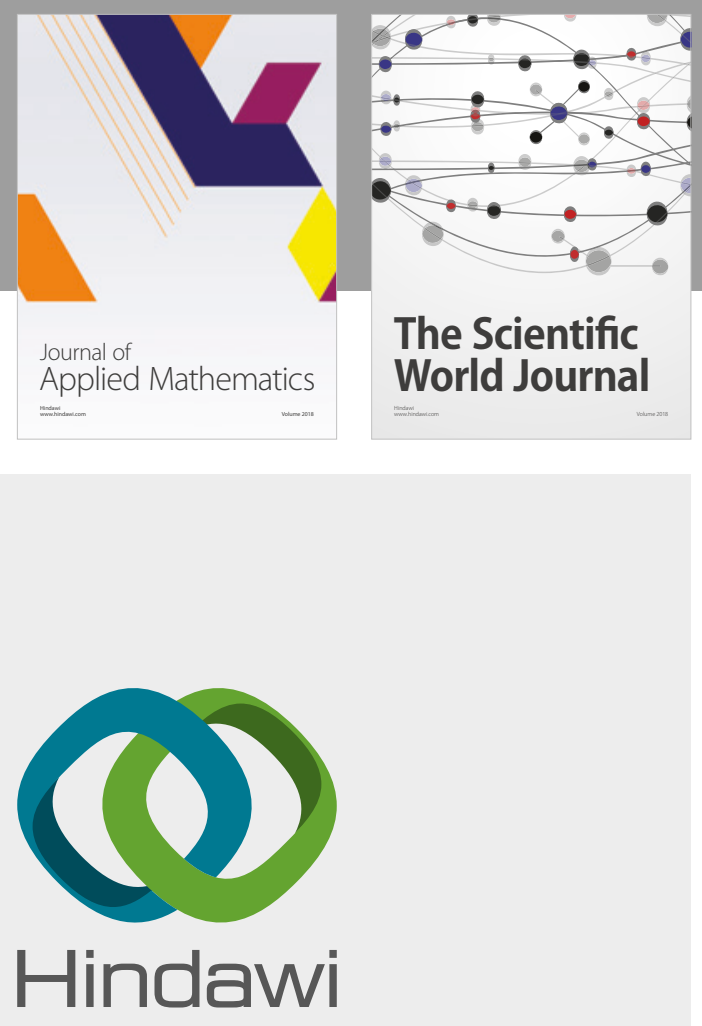

Submit your manuscripts at

www.hindawi.com

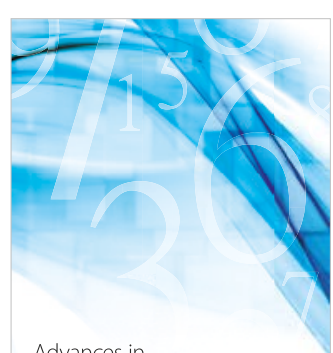

Advances in
Numerical Analysis
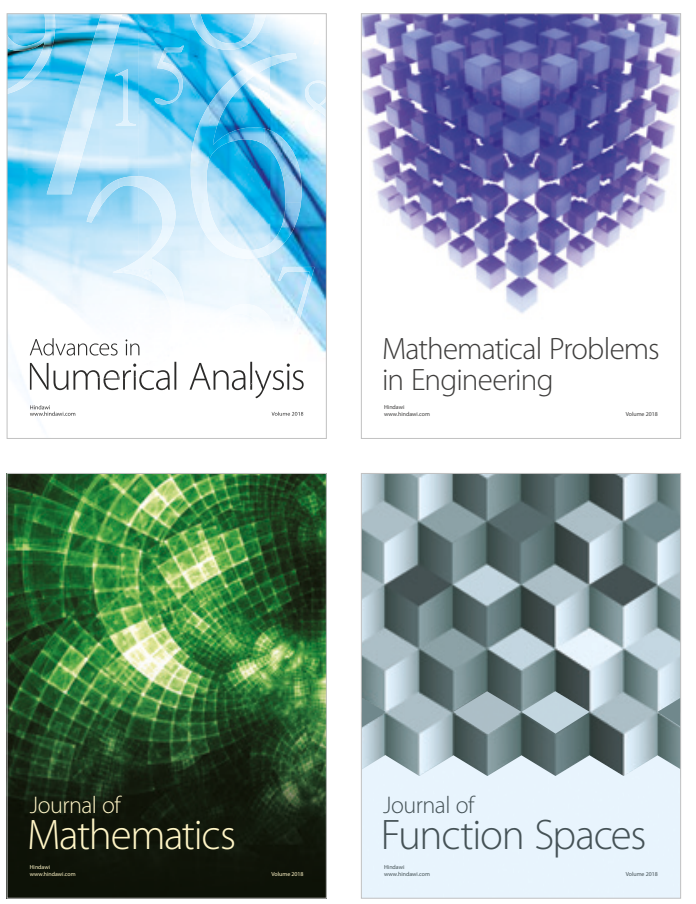

Mathematical Problems in Engineering

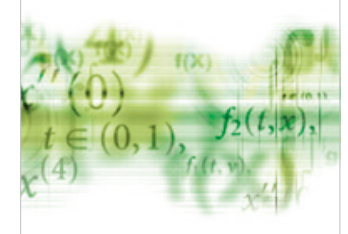

International Journal of

Differential Equations

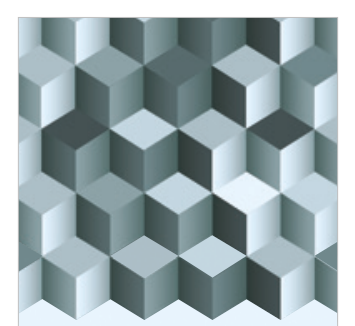

Journal of

Function Spaces
The Scientific

World Journal

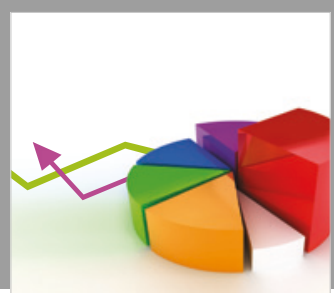

Journal of

Probability and Statistics
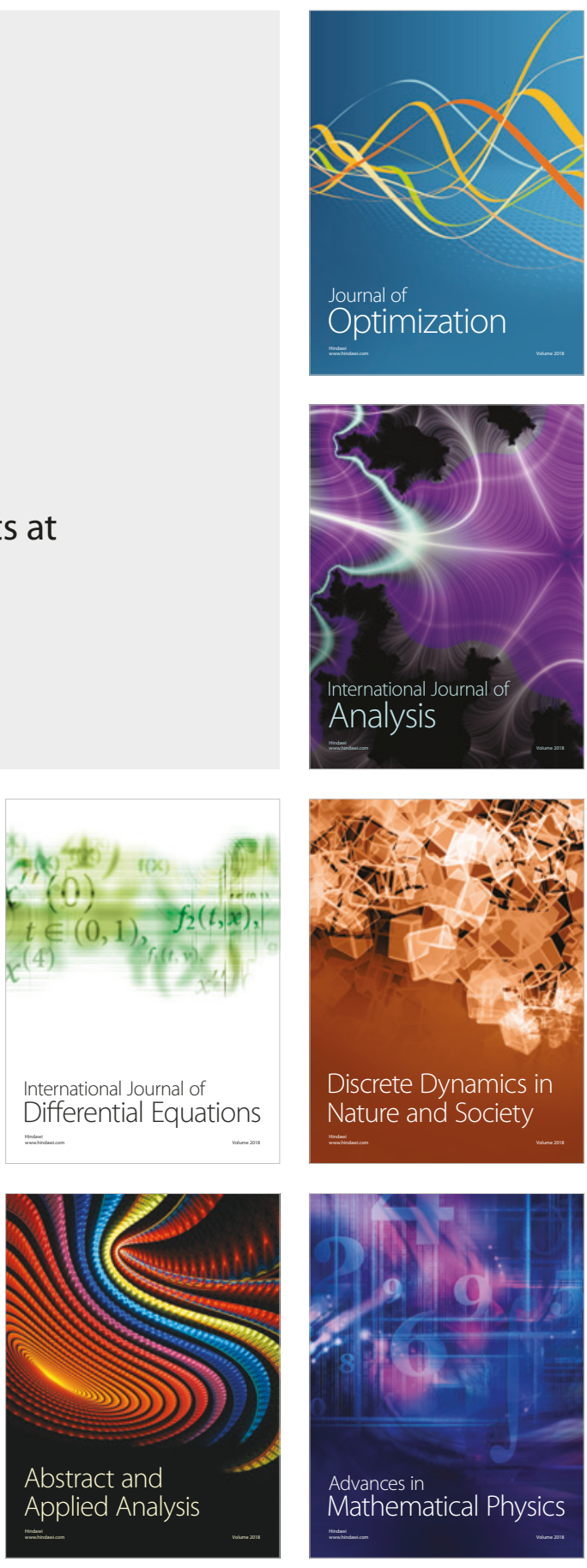